\title{
Is insulin the preferred treatment in persons with type 2 diabetes and liver cirrhosis?
}

\author{
Fu-Shun Yen', Jung-Nien Lai ${ }^{2,3}$, James Cheng-Chung Wei ${ }^{4,5,6}$, Lu-Ting Chiu'7,8, Chih-Cheng Hsu 9,10,11, \\ Ming-Chih Hou ${ }^{12,13^{*}}$ and Chii-Min Hwu $u^{12,14^{*}}$
}

\begin{abstract}
Background: Insulin is highly recommended for diabetes management in persons with liver cirrhosis. However, few studies have evaluated its long-term effects in these persons. We conducted this study to compare the risks of mortality, liver-related complications, and cardiovascular events in persons with type 2 diabetes mellitus (T2DM) and compensated liver cirrhosis.

Methods: From January 1, 2000, to December 31, 2012, we selected 2047 insulin users and 4094 propensity scorematched nonusers from Taiwan's National Health Insurance Research Database. Cox proportional hazard models were used to assess the risks of outcomes.

Results: The mean follow-up time was 5.84 years. The death rate during the follow-up period was 5.28 and 4.07 per 100 person-years for insulin users and nonusers, respectively. In insulin users, the hazard ratios and 95\% confidence intervals (Cls) of all-cause mortality, hepatocellular carcinoma, decompensated cirrhosis, hepatic failure, major cardiovascular events, and hypoglycemia were 1.31 (1.18-1.45), 1.18 (1.05-1.34), 1.53 (1.35-1.72), $1.26(1.42-1.86), 1.41$ (1.23-1.62), and 3.33 (2.45-4.53), respectively.

Conclusions: This retrospective cohort study indicated that among persons with T2DM and compensated liver cirrhosis, insulin users were associated with higher risks of death, liver-related complications, cardiovascular events, and hypoglycemia compared with insulin nonusers.
\end{abstract}

Keywords: All-cause mortality, Hepatocellular carcinoma, Hepatic failure, Decompensated cirrhosis, Hypoglycemia

\section{Background}

Insulin has saved numerous lives since its discovery in the 1920s. It is extremely effective in treating hyperglycemia and can be used when hyperglycemia causes critical conditions, such as ketoacidosis or hyperosmolar hyperglycemic state [1]. By carefully manipulating the dose, insulin is also frequently used in persons with hospitalization, major surgery, sepsis, and acute myocardial infarction [2]. Liver cirrhosis also is the strong indication

\footnotetext{
*Correspondence: mchou@vghtpe.gov.tw; chhwu@vghtpe.gov.tw

${ }^{12}$ Faculty of Medicine, National Yang-Ming University School of Medicine, No. 155, Sec. 2, Linong Street, Taipei 11221, Taiwan

Full list of author information is available at the end of the article
}

for insulin treatment in persons with type 2 diabetes mellitus (T2DM) $[1,2]$.

Liver cirrhosis is an advanced liver disease; it also is the late stage of chronic liver injury [3]. It can be attributed to several reasons, such as nonalcoholic fatty liver diseases, chronic alcoholism, hepatitis B virus (HBV) infection, or hepatitis $\mathrm{C}$ virus (HCV) infection [3]. With the development of cirrhosis, owing to reduced insulin extraction of liver and portal-systemic shunting, serum insulin levels will increase and insulin resistance may develop. Approximately $96 \%$ of persons with cirrhosis may be glucose intolerant and 30\% of them may develop clinical diabetes [4]. Moreover, diabetes treatment in persons with liver cirrhosis is complex [5]. Diet control for 
persons with cirrhosis is not feasible because they may have poor appetite. Encouraging them to exercise may not be suitable because they may demonstrate weakness. Medications such as metformin, sulphonylureas, and thiazolidinedione may cause lactic acidosis (especially in those with chronic alcoholism), may lead to the risk of hypoglycemia, and may aggravate fluid retention, respectively. Thus, adequate management of T2DM in persons with liver cirrhosis is unclear.

Careful adjustment of the insulin dose and close monitoring of blood glucose levels may enable the effective and safe use of insulin for treating persons with cirrhosis and T2DM [6]. However, insulin has some deleterious side effects. Hypoglycemia is the most critical side effect of insulin use, as it can increase the risks of mortality and cardiovascular diseases [7]. Insulin was also reported to increase body weight and risks of cardiovascular events $[8,9]$, and insulin use is associated with the risk of hepatocellular carcinoma (HCC) [10]. Therefore, we conducted this retrospective cohort study to investigate the long-term outcomes of insulin use in people with T2DM and compensated liver cirrhosis.

\section{Methods}

\section{Participants}

This study recruited persons with new diagnoses of T2DM and liver cirrhosis from the Longitudinal Cohort of Diabetes Patients (LHDB) between January 1, 2000, and December 31, 2012, and they were followed until December 31, 2013. LHDB is part of the National Health Insurance (NHI) Research Database (NHIRD). It comprises data of 1,700,000 randomly selected newly diagnosed T2DM patients with longitudinally linked data available from 1997 to 2013. The NHIRD includes the health records of insured persons in Taiwan's NHI program, which was established in 1995 and covered approximately 99\% of Taiwan's 23 million people by 2000 [11]. This administrative database contains information of age, birth date, sex, living areas, treatments, and disease diagnoses according to International Classification of Diseases, Ninth Revision, Clinical Modification (ICD-9-CM) codes. This study was conducted in accordance with the Declaration of Helsinki. To protect personal privacy, all information on the care providers or patients was scrambled before being released. This study was approved by the Research Ethics Committee of China Medical University and Hospital (CMUH104-REC2-115), and the need for informed consent was waived.

\section{Study design}

T2DM was diagnosed based on ICD-9-CM code 250.xx with at least 2 outpatient claims within 1 year or one hospitalization. Persons with ICD-9-CM code 571.5, 571.2, or 571.6 for at least 2 outpatient claims within 1 year or one hospitalization were defined as having liver cirrhosis. This method of defining T2DM and liver cirrhosis using ICD-9-CM codes has been validated by studies $[12,13]$; the diagnostic accuracy of diabetes and cirrhosis is $74.6 \%$ and $82.6 \%$, respectively. Persons with liver cirrhosis and esophageal varices with bleeding (456.0 and 456.2), ascites (789.59 and 789.5), hepatic encephalopathy (572.2), or jaundice (782.4) were defined as having decompensated liver cirrhosis [14] and were initially excluded from this study. Patients without these cirrhotic complications were defined as having compensated liver cirrhosis. We excluded persons who were diagnosed as having type 1 diabetes mellitus (ICD-9-CM code 250.1); who did not receive antidiabetic medications; who were younger than 18 years or older than 80 years; who lacked gender information; who died or had renal failure, stroke, ischemic heart disease, heart failure, HCC, esophageal varices with bleeding, ascites, hepatic encephalopathy, jaundice, or hepatic failure before the index date or within 6 months after the index date; and who were diagnosed as having T2DM or cirrhosis during 1997-1999.

\section{Procedures}

The day of concomitant diagnosis of liver cirrhosis and diabetes was defined as the comorbid date. Persons who underwent insulin therapy for at least 28 days after the comorbid date were defined as insulin users, and those who never took insulin during the whole study period were defined as insulin nonusers. We defined the first date of insulin use as the index date. Variables considered as potential confounders in this study included age, sex, age at the diagnosis of diabetes, the duration of diabetes, antihypertensive and antidiabetic medications, statins, and aspirin. Comorbidities status before the index date included HCV (ICD-9-CM codes 070.41, 070.44, 070.51, 070.54, 070.70, 070.71, and V02.62) and HBV infections (ICD-9-CM codes 070.2, 070.3, and V02.61). We also calculated the Charlson comorbidity index (CCI; a weighted index that consider the number and seriousness of comorbid heart, vascular, chronic pulmonary, connective tissue, mild and moderate liver, ulcer disease, diabetes and related complication, any original or metastatic disease, and AIDS) [15] and Diabetes Complication Severity Index (DCSI; including cardiovascular disease, nephropathy, retinopathy, peripheral vascular disease, stroke, neuropathy, and metabolic diabetes complications) scores [16] to assess the severity of diabetes.

\section{Main outcomes}

We investigated the outcomes of all-cause mortality, major adverse cardiovascular events (MACE), HCC, decompensated cirrhosis, hepatic failure, and 
hypoglycemia. Death was defined as being discharged from the hospital with a death certificate (discharge date was defined as the death date) or termination of NHI coverage after being discharged from hospital due to a critical illness and no further healthcare use for more than 1 year (the end of NHI coverage was defined as the death date). We calculated the incidence rate of MACE, including ischemic heart disease (410-414), stroke (430437), and heart failure (428); HCC (155.x); decompensated cirrhosis (the composite of esophageal varices with bleeding, ascites, hepatic encephalopathy, and jaundice); variceal bleeding; ascites; hepatic encephalopathy; and hepatic failure $(570,572.2,572.4$, and 572.8$)$, to evaluate liver-related complications. We also investigated the incidence of emergency department visited or admitted hypoglycemia $(251.0 x, 251.1 x$, or $251.2 x)$ to evaluate the probable complications of treatments.

\section{Statistical analyses}

Propensity score matching was adopted to optimize comparability between insulin users and nonusers [17]. The propensity score was estimated for every person through nonparsimonious multivariable logistic regression, with insulin treatment as the dependent variable. We used 26 clinically related variables in the analysis as controlling variables (Table 1). The nearest-neighbor algorithm was adopted to construct matching pairs under the assumption that the proportion of $0.995-1.0$ was perfect [18].

Crude and multivariate-adjusted Cox proportional hazard models with robust sandwich standard error estimates were used to compare the risk of outcomes between insulin users and nonusers. The results are presented as hazard ratios (HRs) with 95\% confidence intervals (CIs) for insulin users versus nonusers. To assess the risk of all-cause mortality, we checked the persons' time of death or the end of the study, whichever occurred first. For other outcomes, we checked the persons' date of respective outcomes or end of follow-up on December 31, 2013, whichever occurred first. We compared the cumulative incidence of all-cause mortality, MACE, decompensated cirrhosis, and hepatic failure over time between insulin users and nonusers using the KaplanMeier method and log-rank tests.

We conducted a sensitivity test by excluding persons with hypoglycemia before or after the index date; matching insulin users and nonusers; and calculating the incidence and hazard ratio of death, MACE, and liver-related outcomes to avoid the interference from hypoglycemia on other main outcomes.

Two-tailed $P<0.05$ was considered as significant. SAS v9.4 (SAS Institute, Inc., Cary, NC, USA) was used for the analyses.

\section{Results}

\section{Participants}

From January 1, 2000, to December 31, 2012, a total of 36,853 persons were diagnosed as having T2DM with compensated cirrhosis and were undergoing anti-diabetes treatment. After exclusion of ineligible cases, 2047 persons received insulin treatment for at least 28 days, and 17,173 persons had never received insulin during the follow-up period. Figure 1 depicts the flowchart of patient selection for this study.

Before matching, insulin users presented higher proportions of young age, young ages at diabetes diagnosis, medications use, hepatitis infection than insulin nonusers. People with high CCI and DCSI scores were found among insulin users than among insulin nonusers (Table 1). After propensity score matching, 2047 insulin users and 4094 insulin nonusers were selected. Of insulin users, 1650 (80.61\%), 1289 (62.97\%), and 1781 (87.01\%) persons used basal insulin, premixed insulin, and prandial insulin, respectively. The matched patients are similar in all variables. The mean age of this cohort was 55.09 years, the mean duration of diabetes was 3.13 years, and the HBV and HCV infection rates were 23.33\% and $19.76 \%$, respectively. The mean follow-up time was 5.79 years for insulin users and 5.88 years for nonusers.

\section{Risks of outcomes}

In the matched cohort of people with T2DM and compensated liver cirrhosis, 627 (30.63\%) insulin users and 979 (23.91\%) insulin nonusers died during the follow-up period (incidence rate of 5.28 vs 4.07 per 100 patientyears, respectively). The multivariable-adjusted HR ( $95 \%$ CI) of insulin users to nonusers was 1.31 (1.18-1.45; Table 2).

Table 2 shows that insulin users associated with higher risks of HCC (adjusted hazard ratio [aHR] [95\% CI]: 1.18 [1.05-1.34]), decompensated cirrhosis (aHR [95\% CI]: 1.53 [1.35-1.72]), esophageal varices with bleeding (aHR [95\% CI]: 1.81 [1.16-2.83]), hepatic ascites (aHR [95\% CI]: 1.68 [1.45-1.95]), hepatic encephalopathy (aHR [95\% $\mathrm{CI}]: 1.63$ [1.39-1.91]), and hepatic failure (aHR [95\% CI]: 1.26 [1.42-1.86]) than nonusers; however, insulin users showed no significant difference in the risk of jaundice (aHR [95\% CI]: 0.90 [0.63-1.29]).

Table 2 also displays that insulin users had significantly higher risks of MACE (aHR [95\% CI]: 1.41 [1.23-1.62]), stroke (aHR [95\% CI]: 1.31 [1.09-1.58]), ischemic heart disease (aHR [95\% CI]: 1.36 [1.09-1.71]), and heart failure (aHR [95\% CI]: 2.18 [1.70-2.80]) than nonusers.

Figure 2 shows the cumulative incidence of all-cause mortality, decompensated cirrhosis, hepatic failure, and MACE of insulin users and nonusers with T2DM and compensated liver cirrhosis. 
Table 1 Baseline characteristics of insulin users and nonusers with diabetes and compensated liver cirrhosis

\begin{tabular}{|c|c|c|c|c|c|c|c|c|c|c|}
\hline \multirow[t]{3}{*}{ Variables } & \multicolumn{5}{|c|}{ Before propensity score match } & \multicolumn{5}{|c|}{ After propensity score match } \\
\hline & \multicolumn{2}{|c|}{$\begin{array}{l}\text { Non-insulin users } \\
(n=17,173)\end{array}$} & \multicolumn{2}{|l|}{$\begin{array}{l}\text { Insulin users } \\
(n=2047)\end{array}$} & \multirow[t]{2}{*}{$p$ value } & \multicolumn{2}{|c|}{$\begin{array}{l}\text { Non-insulin users } \\
(\mathrm{n}=4094)\end{array}$} & \multicolumn{2}{|l|}{$\begin{array}{l}\text { Insulin users } \\
(n=2047)\end{array}$} & \multirow[t]{2}{*}{$p$ value } \\
\hline & $\mathrm{N}$ & $\%$ & $\mathbf{N}$ & $\%$ & & $\mathrm{n}$ & $\%$ & $\mathrm{n}$ & $\%$ & \\
\hline Age & & & & & 0.01 & & & & & 0.54 \\
\hline $18-49$ & 5886 & 34.27 & 732 & 35.76 & & 1444 & 35.27 & 732 & 35.76 & \\
\hline $50-65$ & 7301 & 42.51 & 900 & 43.97 & & 1793 & 43.80 & 900 & 43.97 & \\
\hline$>65$ & 3986 & 23.22 & 415 & 20.27 & & 857 & 20.93 & 415 & 20.27 & \\
\hline Mean \pm SD & $55.37 \pm 11.91$ & & $54.98 \pm 11.32$ & & 0.15 & $55.20 \pm 11.82$ & & $54.98 \pm 11.32$ & & 0.29 \\
\hline Sex & & & & & 0.11 & & & & & 0.62 \\
\hline Female & 5421 & 31.57 & 611 & 29.85 & & 1197 & 29.24 & 611 & 29.85 & \\
\hline Male & 11,752 & 68.43 & 1436 & 70.15 & & 2897 & 70.76 & 1436 & 70.15 & \\
\hline $\mathrm{DM}$ age, mean $\pm S D$ & $55.96 \pm 11.12$ & & $51.93 \pm 10.93$ & & $<0.0001$ & $52.58 \pm 11.03$ & & $51.93 \pm 10.93$ & & 0.06 \\
\hline $\mathrm{DM}$ duration, mean $\pm \mathrm{SD}^{\mathrm{a}}$ & $4.85 \pm 3.47$ & & $3.05 \pm 2.79$ & & $<0.0001$ & $3.21 \pm 3.94$ & & $3.05 \pm 2.79$ & & 0.10 \\
\hline \multicolumn{11}{|l|}{ Antihypertensive drugs } \\
\hline ACEI/ARB & 7401 & 43.10 & 1076 & 52.56 & $<0.0001$ & 2143 & 52.34 & 1076 & 52.56 & 0.87 \\
\hline$\beta$-blockers & 9004 & 52.43 & 1298 & 63.41 & $<0.0001$ & 2567 & 62.70 & 1298 & 63.41 & 0.59 \\
\hline Calcium-channel blockers & 5086 & 29.62 & 777 & 37.96 & $<0.0001$ & 1495 & 36.52 & 777 & 37.96 & 0.27 \\
\hline Diuretics & 4304 & 25.06 & 699 & 34.15 & $<0.0001$ & 1384 & 33.81 & 699 & 34.15 & 0.79 \\
\hline Other anti-hypertensive agent & 3350 & 19.51 & 521 & 25.45 & $<0.0001$ & 1034 & 25.26 & 521 & 25.45 & 0.87 \\
\hline \multicolumn{11}{|l|}{ Antidiabetic drugs } \\
\hline Metformin & 5437 & 31.66 & 1033 & 50.46 & $<0.0001$ & 2036 & 49.73 & 1033 & 50.46 & 0.59 \\
\hline Sulfonylurea & 6446 & 37.54 & 1178 & 57.55 & $<0.0001$ & 2337 & 57.08 & 1178 & 57.55 & 0.73 \\
\hline Meglitinide & 1814 & 10.56 & 327 & 15.97 & $<0.0001$ & 644 & 15.73 & 327 & 15.97 & 0.80 \\
\hline Thiazolidinedione & 2050 & 11.94 & 429 & 20.96 & $<0.0001$ & 789 & 19.27 & 429 & 20.96 & 0.12 \\
\hline a-glucosidase inhibitor & 1779 & 10.36 & 341 & 16.66 & $<0.0001$ & 644 & 15.73 & 341 & 16.66 & 0.35 \\
\hline DPP-4 inhibitors & 457 & 2.66 & 92 & 4.49 & $<0.0001$ & 199 & 4.86 & 92 & 4.49 & 0.53 \\
\hline \multicolumn{11}{|l|}{ Other drugs } \\
\hline Statin & 4202 & 24.47 & 661 & 32.29 & $<0.0001$ & 1299 & 31.73 & 661 & 32.29 & 0.66 \\
\hline Aspirin & 9556 & 55.65 & 1354 & 66.15 & $<0.0001$ & 2762 & 67.46 & 1354 & 66.15 & 0.30 \\
\hline DCSI score & & & & & $<0.0001$ & & & & & 0.59 \\
\hline 0 & 9298 & 54.14 & 732 & 35.76 & & 1418 & 34.64 & 732 & 35.76 & \\
\hline 1 & 2595 & 15.11 & 397 & 19.39 & & 786 & 19.20 & 397 & 19.39 & \\
\hline$\geq 2$ & 5280 & 30.75 & 918 & 44.85 & & 1890 & 46.17 & 918 & 44.85 & \\
\hline $\mathrm{CCl}$ & & & & & $<0.0001$ & & & & & 0.64 \\
\hline 0 & 11,657 & 67.88 & 694 & 33.90 & & 1357 & 33.15 & 694 & 33.90 & \\
\hline 1 & 2326 & 13.54 & 528 & 25.79 & & 1101 & 26.89 & 528 & 25.79 & \\
\hline$\geq 2$ & 3190 & 18.58 & 825 & 40.31 & $<0.0001$ & 1636 & 39.96 & 825 & 40.30 & \\
\hline HBV & 2810 & 16.36 & 491 & 23.99 & $<0.0001$ & 928 & 22.67 & 491 & 23.99 & 0.25 \\
\hline $\mathrm{HCV}$ & 2093 & 12.19 & 407 & 19.88 & $<0.0001$ & 804 & 19.64 & 407 & 19.88 & 0.82 \\
\hline
\end{tabular}

SD, standard deviation; ACEI, angiotensin converting enzyme inhibitor; ARB, angiotensin receptor blocker; CCl, Charlson comorbidity index; DCSI score, diabetes complications severity index score; $\mathrm{HBV}$, hepatitis $B$ virus; $\mathrm{HCV}$, hepatitis $C$ virus

a $t$-test

Insulin users had a higher risk of hypoglycemia (aHR [95\% CI]: 3.33 [2.45-4.53]) than nonusers (Table 2).

\section{Sensitivity analysis}

Table 3 presents the results of the sensitivity analysis of all-cause mortality, liver-related outcomes, and
MACE, in which persons with hypoglycemia before and during the follow-up periods were excluded. Insulin users showed higher risks of all-cause mortality, HCC, decompensated cirrhosis, hepatic failure, and MACE than nonusers. 


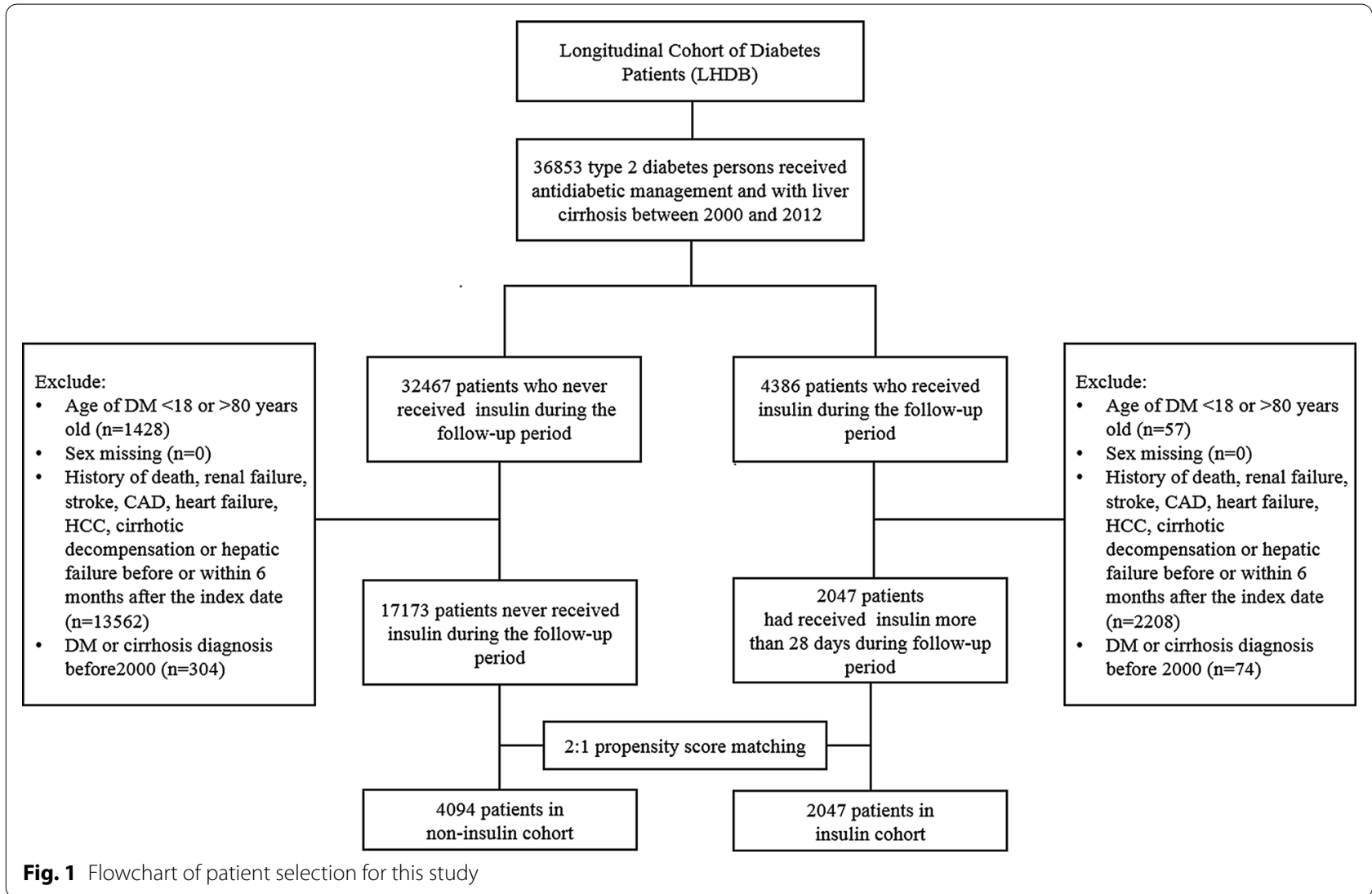

Table 2 Outcomes of insulin users and matched nonusers with diabetes and compensated liver cirrhosis

\begin{tabular}{|c|c|c|c|c|c|c|c|c|c|c|}
\hline \multirow[t]{2}{*}{ Outcomes } & \multicolumn{3}{|c|}{$\begin{array}{l}\text { Non-insulin users } \\
(n=4094)\end{array}$} & \multicolumn{3}{|c|}{$\begin{array}{l}\text { Insulin users } \\
(\mathrm{n}=2047)\end{array}$} & \multirow[t]{2}{*}{ Crude HR $(95 \% \mathrm{Cl})$} & \multirow[t]{2}{*}{$p$ value } & \multirow[t]{2}{*}{ Adjusted $\mathrm{HR}^{\mathrm{a}}(95 \% \mathrm{Cl})$} & \multirow[t]{2}{*}{$p$ value } \\
\hline & Events & PY & IR & Events & PY & IR & & & & \\
\hline All-cause mortality & 979 & 24,075 & 4.07 & 627 & 11,866 & 5.28 & $1.33(1.21-1.48)$ & $<0.0001$ & $1.31(1.18-1.45)$ & $<0.0001$ \\
\hline $\mathrm{HCC}$ & 700 & 22,490 & 3.11 & 402 & 10,921 & 3.68 & $1.17(1.03-1.32)$ & 0.01 & $1.18(1.05-1.34)$ & 0.007 \\
\hline MACE & 512 & 22,227 & 2.30 & 339 & 10,738 & 3.16 & $1.37(1.19-1.57)$ & $<0.0001$ & $1.41(1.23-1.62)$ & $<0.0001$ \\
\hline Stroke & 300 & 23,086 & 1.30 & 186 & 11,285 & 1.65 & $1.28(1.07-1.54)$ & 0.007 & $1.31(1.09-1.58)$ & 0.004 \\
\hline Ischemic heart disease & 198 & 23,277 & 0.85 & 128 & 11,394 & 1.12 & $1.31(1.05-1.64)$ & 0.02 & $1.36(1.09-1.71)$ & 0.006 \\
\hline Heart failure & 125 & 23,649 & 0.53 & 128 & 11,507 & 1.11 & $2.11(1.65-2.71)$ & $<0.0001$ & $2.18(1.70-2.80)$ & $<0.0001$ \\
\hline Decompensated cirrhosis & 642 & 22,865 & 2.81 & 465 & 10,890 & 4.27 & $1.50(1.33-1.69)$ & $<0.0001$ & $1.53(1.35-1.72)$ & $<0.0001$ \\
\hline Variceal bleeding & 41 & 23,986 & 0.17 & 38 & 11,767 & 0.32 & $1.83(1.18-2.85)$ & 0.007 & $1.81(1.16-2.83)$ & 0.009 \\
\hline Hepatic ascites & 407 & 23,381 & 1.74 & 327 & 11,196 & 2.92 & $1.66(1.43-1.92)$ & $<0.0001$ & $1.68(1.45-1.95)$ & $<0.0001$ \\
\hline Hepatic encephalopathy & 351 & 23,577 & 1.49 & 281 & 11,380 & 2.47 & $1.64(1.40-1.91)$ & $<0.0001$ & $1.63(1.39-1.91)$ & $<0.0001$ \\
\hline Jaundice & 102 & 23,848 & 0.43 & 45 & 11,757 & 0.38 & $0.88(0.62-1.25)$ & 0.49 & $0.90(0.63-1.29)$ & 0.58 \\
\hline Hepatic failure & 493 & 23,363 & 2.11 & 388 & 11,230 & 3.46 & $1.62(1.42-1.85)$ & $<0.0001$ & $1.26(1.42-1.86)$ & $<0.0001$ \\
\hline Hypoglycemia & 68 & 23,893 & 0.28 & 107 & 11,576 & 0.92 & $3.26(2.40-4.42)$ & $<0.0001$ & $3.33(2.45-4.53)$ & $<0.0001$ \\
\hline
\end{tabular}

$\mathrm{PY}$, person-years; IR, incidence rate, per 100 person-years; $\mathrm{HR}$, hazard ratio; $\mathrm{Cl}$, confidence interval; $\mathrm{HCC}$, hepatocellular carcinoma; $\mathrm{MACE}$, major adverse cardiac event, including stroke, ischemic heart disease, and heart failure

${ }^{a}$ Adjusted for age, sex, index year, age of diabetes mellitus diagnosis, DM duration (years), antihypertensive drugs (ACE inhibitors, ARBs, $\beta$-blockers, calcium-channel blockers, diuretics, other antihypertensive), antidiabetic drugs (metformin, sulfonylureas, meglitinides, TZD, a-glucosidase inhibitor, DPP-4 inhibitors), statin, and aspirin, $\mathrm{CCl}(0,1, \geq 2)$, DCSI score $(0,1, \geq 2)$, HBV and HCV 

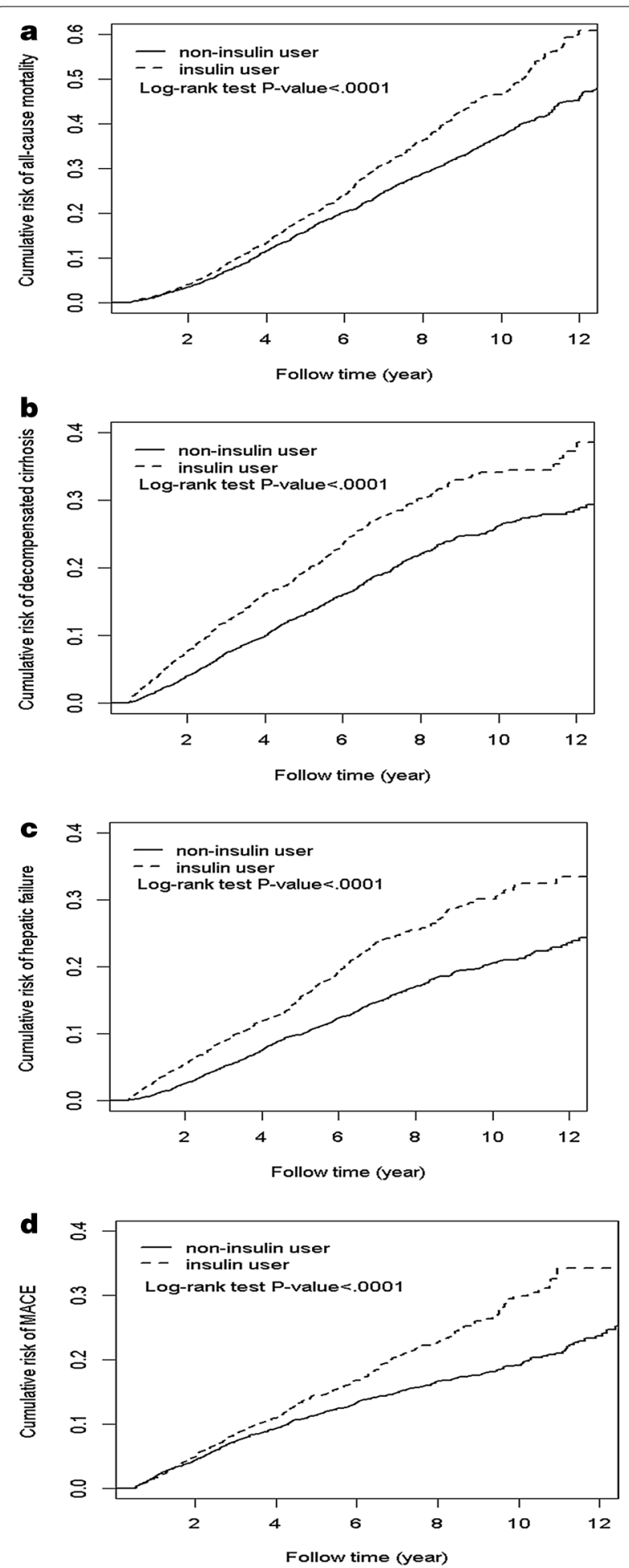

Fig. 2 Cumulative incidence curves of four outcomes between insulin users and nonusers

\section{Discussion}

Our study indicated that in people with T2DM and compensated cirrhosis, insulin users showed higher risks of all-cause mortality, cardiovascular events, HCC, decompensated cirrhosis, hepatic failure, and hypoglycemia than insulin nonusers, even after excluding persons with hypoglycemia.

Insulin treatment is frequently used in persons with diabetes and liver cirrhosis. Elkrief et al. reported that of 348 persons with hepatitis C-related cirrhosis, $62 \%$ were on insulin therapy [19]. Gentile et al. found that acarbose significantly improved fasting and postprandial glucose levels in 100 persons with compensated cirrhosis and insulin-treated T2DM [20]. They also compared the metabolic profiles of lispro and regular human insulin in persons with diet-unresponsive T2DM and compensated nonalcoholic liver disease and found that lispro caused lower postprandial glucose levels and hypoglycemic rates [6]. Insulin requirements in persons with liver cirrhosis vary; persons with decompensated cirrhosis may need less insulin compared with persons diagnosed as having compensated cirrhosis [5]. Therefore, insulin therapy in people with liver cirrhosis requires close monitoring of blood glucose levels to avoid the risks of hypoglycemia or hyperglycemia. Our study disclosed that the use of insulin was associated with a significantly higher risk of severe hypoglycemia in persons with compensated cirrhosis compared with oral antidiabetic agents.

People with liver cirrhosis have a 5-10 times higher risk of death than the general population [21], and diabetes can increase their mortality risk [22]. Insulin was reported to be associated with a high risk of mortality in persons with T2DM [23]; our study also showed that among persons with compensated liver cirrhosis, insulin users demonstrated a higher risk of all-cause mortality than insulin nonusers. Although hypoglycemia may increase the risk of death, we observed similar results even after excluding persons with hypoglycemia. Moreover, because insulin users in this study showed increased risks of major cardiovascular events, cirrhotic decompensation, and liver failure, these conditions may also increase the risk of death.

People with liver cirrhosis were reported to have a low prevalence of cardiovascular diseases [24], which may be because of their short life expectancy and low levels of clotting factors in their blood. Coexisting T2DM may increase the risk of cardiovascular diseases; however, their prevalence is still lower than that of general population with T2DM only [24]. Insulin therapy was reported 
Table 3 Outcomes of insulin users and matched nonusers after excluding persons with hypoglycemia

\begin{tabular}{|c|c|c|c|c|c|c|c|c|c|c|}
\hline \multirow[t]{2}{*}{ Outcomes } & \multicolumn{3}{|c|}{$\begin{array}{l}\text { Non-insulin users } \\
(n=4026)\end{array}$} & \multicolumn{3}{|c|}{$\begin{array}{l}\text { Insulin users } \\
(n=1940)\end{array}$} & \multirow[t]{2}{*}{ Crude HR (95\% Cl) } & \multirow[t]{2}{*}{$p$ value } & \multirow[t]{2}{*}{ Adjusted $\mathrm{HR}^{\mathrm{a}}(95 \% \mathrm{Cl})$} & \multirow[t]{2}{*}{$p$ value } \\
\hline & Events & PY & IR & Events & PY & IR & & & & \\
\hline All-cause mortality & 951 & 23,577 & 4.03 & 575 & 11,131 & 5.17 & $1.32(1.19-1.47)$ & $<0.0001$ & $1.30(1.17-1.44)$ & $<0.0001$ \\
\hline $\mathrm{HCC}$ & 682 & 22,041 & 3.09 & 386 & 10,219 & 3.78 & $1.21(1.07-1.37)$ & 0.003 & $1.22(1.08-1.39)$ & 0.002 \\
\hline MACE & 490 & 21,816 & 2.25 & 303 & 10,152 & 2.98 & $1.33(1.15-1.54)$ & $<0.0001$ & $1.37(1.19-1.58)$ & $<0.0001$ \\
\hline Stroke & 289 & 22,616 & 1.28 & 165 & 10,620 & 1.55 & $1.23(1.01-1.48)$ & 0.04 & $1.25(1.04-1.53)$ & 0.02 \\
\hline Ischemic heart disease & 188 & 22,819 & 0.82 & 111 & 10,727 & 1.03 & $1.25(0.99-1.59)$ & 0.06 & $1.32(1.04-1.68)$ & 0.02 \\
\hline Heart failure & 118 & 23,187 & 0.51 & 112 & 10,841 & 1.03 & $2.04(1.57-2.64)$ & $<0.0001$ & $2.12(1.63-2.75)$ & $<0.0001$ \\
\hline Decompensated cirrhosis & 626 & 22,411 & 2.79 & 432 & 10,219 & 4.23 & $1.50(1.33-1.69)$ & $<0.0001$ & $1.52(1.34-1.72)$ & $<0.0001$ \\
\hline Esophageal varicose & 41 & 23,488 & 0.17 & 37 & 11,034 & 0.34 & $1.86(1.20-2.91)$ & 0.006 & $1.86(1.19-1.92)$ & 0.007 \\
\hline Hepatic ascites & 395 & 22,915 & 1.72 & 298 & 10,518 & 2.83 & $1.62(1.40-1.89)$ & $<0.0001$ & $1.65(1.42-1.92)$ & $<0.0001$ \\
\hline Hepatic encephalopathy & 339 & 23,105 & 1.47 & 265 & 10,672 & 2.48 & $1.67(1.52-1.96)$ & $<0.0001$ & $1.67(1.42-1.97)$ & $<0.000$ \\
\hline Jaundice & 101 & 23,355 & 0.43 & 44 & 11,027 & 0.40 & $0.91(0.64-1.30)$ & 0.61 & $0.95(0.66-1.36)$ & 0.78 \\
\hline Hepatic failure & 481 & 22,891 & 2.10 & 362 & 10,528 & 3.44 & $1.62(1.42-1.87)$ & $<0.0001$ & $1.63(1.42-1.88)$ & $<0.0001$ \\
\hline
\end{tabular}

PY, person-years; IR, incidence rate, per 100 person-years; $\mathrm{HR}$, hazard ratio; $\mathrm{Cl}$, confidence interval; $\mathrm{HCC}$, hepatocellular carcinoma; MACE, major adverse cardiac event, including stroke, ischemic heart disease, and heart failure

${ }^{a}$ Adjusted for age, sex, index year, DM age, DM duration (years), antihypertensive drugs (ACE inhibitors, ARBs, $\beta$-blockers, calcium-channel blockers, diuretics, other antihypertensive), antidiabetic drugs (metformin, sulfonylureas, meglitinides, TZD, a-glucosidase inhibitor, DPP-4 inhibitors), statins, and aspirin, CIC index (0, 1, $\geq 2$ ), DCSI score $(0,1, \geq 2), \mathrm{HBV}$ and HCV

to increase the risk of cardiovascular complications in persons with T2DM [8]. Our study also illustrated that insulin use in persons with compensated liver cirrhosis was associated with a higher risk of MACE, and these hazards persisted even after excluding persons with hypoglycemia. Excess exposure to insulin and hyperinsulinemia are thought to increase basal insulin signaling, which can contribute to insulin resistance and cause atherosclerosis [25].

T2DM $[5,19,26]$ and suboptimal glycemic levels [26] in persons with liver cirrhosis were reported to increase the risks of liver-related complications. However, the favorable impact of optimal glycemic management in persons with liver cirrhosis has not been demonstrated yet. Our study compared the progression of cirrhotic complications between insulin users and nonusers with compensated cirrhosis and observed that insulin users seemed to have higher risks of variceal bleeding, ascites, hepatic encephalopathy, and hepatic failure than insulin nonusers. Insulin stimulates adrenergic hormones and releases endothelin-1 [27]. It was reported to have vasoconstrictor effects on isolated arterioles [28], which may increase systemic vascular resistance and portal pressure. Cirrhosis can aggravate insulin resistance and disturb the molecular mechanisms of insulin on hepatocytes. Exogenous insulin and consequent hyperinsulinemia may activate some signaling molecules (such as PHLPP1 and Grb14) and influence hepatocyte apoptosis [29, 30]. These factors may exacerbate the progression of liver cirrhosis and hepatic failure.
HCC occurs primarily in persons with cirrhosis, and diabetes can exacerbate this risk [5]. The use of insulin was reported to increase the risk of $\operatorname{HCC}[10,26]$; our study supports this finding because our results showed that insulin users had a higher risk of HCC than insulin nonusers. Through the activation of the insulin-like growth factor signaling pathway, exogenous insulin and hyperinsulinemia may accelerate hepatocarcinogenesis in persons with liver cirrhosis.

This study has some limitations. First, this was a nationwide cohort study using a sample of Chinese ethnicity only; therefore, the results cannot be generalized to other ethnicities. Second, the administrative claims dataset does not have information on body weight, physical activity, alcohol consumption, and cigarette smoking. It does not contain data on blood biochemical and hemoglobin $\mathrm{A} 1 \mathrm{C}$ results, which are used to assess the severity of liver cirrhosis and the treatment situation of T2DM. Instead, we used clinical diagnoses to divide persons into those with compensated and decompensated liver cirrhosis and used DCSI and diabetes duration to distinguish the severity of T2DM. We performed propensity score matching to balance critical variables between insulin users and nonusers to maximally reduce the bias from known confounders. However, the above mentioned unmeasured factors may affect our results. Third, due to no linkage to the National Death Registry, the definition of death in this study includes patients no longer coverage by NHI after discharge from critical illness; which may overestimate the incidence of mortality. Fourth, 
because the number of insulin pens is counted instead of units of insulin in insulin prescription in our health system, we cannot accurately calculate the doses of insulin used. The patients' adherence to prescribed insulin injections or oral antidiabetic drugs also cannot be adequately measured using this health insurance database. Moreover, physicians may choose insulin therapy according to the severity of patients, this cofounding by indication also needs to be noted. Finally, a cohort study is always subject to some inevitable bias, and randomized controlled studies are warranted to verify our results.

\section{Conclusion}

Although insulin is the recommended treatment for persons with T2DM and liver cirrhosis, few clinical studies have evaluated its long-term effects and safety. In this retrospective cohort study, insulin use in people with T2DM and compensated cirrhosis was associated with higher risks of hypoglycemia, cardiovascular events, liver-related complications, and mortality than insulin nonusers. Therefore, in persons with compensated liver cirrhosis, the use of insulin may require special attention.

\begin{abstract}
Abbreviations
T2DM: Type 2 diabetes mellitus; HBV: Hepatitis B virus; HCV: Hepatitis C virus; HCC: Hepatocellular carcinoma; CCl: Charlson comorbidity index; DCSI: Diabetes complication severity index; MACE: Major adverse cardiovascular events.
\end{abstract}

\section{Acknowledgements}

This manuscript was edited by Wallace Academic Editing.

\section{Authors' contributions}

FSY and CMH participated in the study design. JCW, CCH, and MCH participated in the study coordination and data collection. JNL and CCH participated in the data analysis; all authors contributed to the interpretation of the results and the discussion. FSY, CCH, MCH, and $\mathrm{CMH}$ participated in manuscript writing; all authors participated in revising the manuscript. $\mathrm{MCH}$ and $\mathrm{CMH}$ supervised the study. All authors read and approved the final manuscript.

\section{Funding}

This study was supported in part by the Taiwan Ministry of Health and Welfare Clinical Trial Center (MOHW109-TDU-B-212-114004), MOST Clinical Trial Consortium for Stroke (MOST 108-2321-B-039-003), Tseng-Lien Lin Foundation, Taichung, and Taipei Veterans General Hospital (V105C-204), Taiwan. These funding agencies had no role in the study design, data collection and analysis, decision to publish, or manuscript preparation. The writing and preparation of this paper were not funded by any organization, and data analysis was not undertaken by employees of funders or any author who received the funding. The funders did not offer support for writing. The corresponding authors had complete access to all data in the study and thus the final responsibility for the decision to publish it.

\section{Availability of data and materials}

Data are available from the National Health Insurance Research Database (NHIRD) published by Taiwan National Health Insurance (NHI) Bureau. The data utilized in this study cannot be made available in the paper, the supplemental files, or in a public repository due to the "Personal Information Protection Act" executed by Taiwan's government, starting from 2012. Requests for data can be sent as a formal proposal to the NHIRD (http://nhird.nhri.org.tw) or by email to nhird@nhri.org.tw.

\section{Declarations}

Ethics approval and consent to participate

We confirmed that all methods were performed in accordance to the Declaration of Helsinki. To protect personal privacy, all information on the care providers or patients was scrambled before being released. This study was approved by the Research Ethics Committee of China Medical University and Hospital (CMUH104-REC2-115), and the need for informed consent was waived.

\section{Consent for publication}

Not applicable.

\section{Competing interests}

The authors declare that they have no competing interests.

\section{Author details}

${ }^{1}$ Dr. Yen's Clinic, No. 15, Shanying Road, Gueishan District, Taoyuan 33354, Taiwan. ${ }^{2}$ School of Chinese Medicine, College of Chinese Medicine, China Medical University, No. 91, Hsueh-Shih Road, Taichung 40402, Taiwan. ${ }^{3}$ Department of Chinese Medicine, China Medical University Hospital, 3F., No. 373-2, Jianxing Road, Taichung 40459, Taiwan. ${ }^{4}$ Department of Allergy, Immunology and Rheumatology, Chung Shan Medical University Hospital, Taichung City 40201, Taiwan. ${ }^{5}$ Institute of Medicine, Chung Shan Medical University, Taichung City 40201, Taiwan. ${ }^{6}$ Graduate Institute of Integrated Medicine, China Medical University, No. 110, Sec. 1, Jianguo N. Rd., South District, Taichung City 40201, Taiwan. ${ }^{7}$ Management Office for Health Data, China Medical University Hospital, 3F., No. 373-2, Jianxing Road, Taichung 40459, Taiwan. ${ }^{8}$ College of Medicine, China Medical University, No. 110, Sec. 1, Jianguo N. Rd., South District, Taichung City 40201, Taiwan. ${ }^{9}$ Institute of Population Health Sciences, National Health Research Institutes, 35 Keyan Road, Zhunan, Miaoli County 35053, Taiwan. ${ }^{10}$ Department of Health Services Administration, China Medical University, No. 91, Hsueh-Shih Road, Taichung 40402, Taiwan. ${ }^{11}$ Department of Family Medicine, Min-Sheng General Hospital, 168 ChingKuo Road, Taoyuan 33044, Taiwan. ${ }^{12}$ Faculty of Medicine, National Yang-Ming University School of Medicine, No. 155, Sec. 2, Linong Street, Taipei 11221, Taiwan. ${ }^{13}$ Division of Gastroenterology and Hepatology, Department of Medicine, Taipei Veterans General Hospital, No. 201, Sec. 2, Shipai Road, Beitou District, Taipei 11217, Taiwan. ${ }^{14}$ Section of Endocrinology and Metabolism, Department of Medicine, Taipei Veterans General Hospital, No. 201, Sec. 2, Shipai Road, Beitou District, Taipei 11217, Taiwan.

Received: 28 December 2020 Accepted: 22 March 2021

Published online: 12 June 2021

\section{References}

1. Inzucchi SE, Bergenstal RM, Buse JB, Diamant M, Ferrannini E, Nauck M, et al. Management of hyperglycemia in type 2 diabetes: a patient-centered approach: position statement of the American Diabetes Association (ADA) and the European Association for the Study of Diabetes (EASD). Diabetes Care. 2012;35:1364-79. https://doi.org/10.2337/dc12-0413.

2. Cahn A, Miccoli R, Dardano A, Del Prato S. New forms of insulin and insulin therapies for the treatment of type 2 diabetes. Lancet Diabetes Endocrinol. 2015;3:638-52. https://doi.org/10.1016/S2213-8587(15) 00097-2.

3. Anthony PP, Ishak KG, Nayak NC, Poulsen HE, Scheuer PJ, Sobin LH. The morphology of cirrhosis: recommendations on definition, nomenclature, and classification by a working group sponsored by the World Health Organization. J Clin Pathol. 1978;31:395-414. https://doi.org/10.1136/jcp. 31.5.395.

4. Petrides AS, Vogt C, Schulze-Berge D, Matthews D, Strohmeyer G. Pathogenesis of glucose intolerance and diabetes mellitus in cirrhosis. Hepatology. 1994;19:616-27. https://doi.org/10.1002/hep.1840190312.

5. Elkrief L, Rautou PE, Sarin S, Valla D, Paradis V, Moreau R. Diabetes mellitus in patients with cirrhosis: clinical implications and management. Liver Int. 2016:36:936-48. https://doi.org/10.1111/liv.13115.

6. Gentile S, Guarino G, Strollo F, Romano M, Genovese S, Masarone CA. Lispro insulin in people with non-alcoholic liver cirrhosis and type 2 
diabetes mellitus. Diabetes Res Clin Pract. 2016;113:179-86. https://doi. org/10.1016/j.diabres.2015.12.006.

7. Frier BM, Schernthaner G, Heller SR. Hypoglycemia and cardiovascular risks. Diabetes Care. 2011;34:S132-7. https://doi.org/10.2337/dc11-s220.

8. Herman ME, O'Keefe JH, Bell DSH, Schwartz SS. Insulin therapy increases cardiovascular risk in type 2 diabetes. Prog Cardiovasc Dis. 2017;60:42234. https://doi.org/10.1016/j.pcad.2017.09.001.

9. Holden SE, Jenkins-Jones S, Morgan CL, Schernthaner G, Currie CJ. Glucose-lowering with exogenous insulin monotherapy in type 2 diabetes: dose association with all-cause mortality, cardiovascular events and cancer. Diabetes Obes Metab. 2015;17:350-62. https://doi.org/10.1111/ dom.12412.

10. Donadon V, Balbi M, Zanette G. Hyperinsulinemia and risk for hepatocellular carcinoma in patients with chronic liver diseases and type 2 diabetes mellitus. Expert Rev Gastroenterol Hepatol. 2009;3:465-7. https://doi.org/ 10.1586/egh.09.41.

11. Cheng TM. Taiwan's new national health insurance program: genesis and experience so far. Health Aff (Millwood). 2003;22:61-76. https://doi.org/ 10.1377/hlthaff.22.3.61.

12. Lin CC, Lai MS, Syu CY, Chang SC, Tseng FY. Accuracy of diabetes diagnosis in health insurance claims data in Taiwan. J Formos Med Assoc. 2005;104:157-63.

13. Nehra MS, Ma Y, Clark C, Amarasingham R, Rockey DC, Singal AG. Use of administrative claims data for identifying patients with cirrhosis. J Clin Gastroenterol. 2013;47:e50-4. https://doi.org/10.1097/MCG.0b013e3182 688d2f.

14. Mukerji AN, Patel V, Jain A. Improving survival in decompensated cirrhosis. Int J Hepatol. 2012;2012:318627. https://doi.org/10.1155/2012/ 318627.

15. Meduru P, Helmer D, Rajan M, Tseng CL, Pogach L, Sambamoorthi U. Chronic illness with complexity: implications for performance measurement of optimal glycemic control. J Gen Intern Med. 2007;22:408-18. https://doi.org/10.1007/s11606-007-0310-5.

16. Young BA, Lin E, Von Korff M, Simon G, Ciechanowski P, Ludman EJ, Everson-Stewart S, et al. Diabetes complications severity index and risk of mortality, hospitalization, and health care utilization. Am J Manag Care. 2008;14:15-23.

17. D'Agostino RB Jr. Propensity score methods for bias reduction in the comparison of a treatment to a non-randomized control group. Stat Med. 1998;17:2265-81. https://doi.org/10.1002/(sici)1097-0258(19981015)17: 19\%3c2265::aid-sim918\%3e3.0.co;2-b.

18. lezzoni LI. Risk adjustment for measuring healthcare outcomes. Chicago: Health Administration Press; 1997.

19. Elkrief L, Chouinard P, Bendersky N, Hajage D, Larroque B, Babanye G, et al. Diabetes mellitus is an independent prognostic factor for major liver related outcomes in patients with cirrhosis and chronic hepatitis $C$ Hepatology. 2014;60:823-31. https://doi.org/10.1002/hep.27228.
20. Gentile S, Turco S, Guarino G, Oliviero B, Annunziata S, Cozzolino D, et al. Effect of treatment with acarbose and insulin in patients with noninsulin-dependent diabetes mellitus associated with non-alcoholic liver cirrhosis. Diabetes Obes Metab. 2001;3:33-40. https://doi.org/10.1046/j. 1463-1326.2001.00103.x.

21. Fleming KM, Aithal GP, Card TR, West J. All-cause mortality in people with cirrhosis compared with the general population: a population-based cohort study. Liver Int. 2012;32:79-84. https://doi.org/10.1111/j.14783231.2011.02517.x

22. Garcia-Compean D, Jaquez-Quintana JO, Gonzalez-Gonzalez JA, Maldonado-Garza H. Liver cirrhosis and diabetes: risk factors, pathophysiology, clinical implications and management. World J Gastroenterol. 2009;15:280-8. https://doi.org/10.3748/wjg.15.280.

23. Gamble JM, Simpson SH, Eurich DT, Majumdar SR, Johnson JA. Insulin use and increased risk of mortality in type 2 diabetes: a cohort study. Diabetes Obes Metab. 2010;12:47-53. https://doi.org/10.1111/j.1463-1326. 2009.01125.x.

24. Marchesini G, Ronchi M, Forlani G, Bugianesi E, Bianchi G, Fabbri A, et al. Cardiovascular disease in cirrhosis - a point-prevalence study in relation to glucose tolerance. Am J Gastroenterol. 1999;94:655-62. https://doi. org/10.1111/j.1572-0241.1999.00931.x.

25. Cao W, Ning J, Yang X, Liu Z. Excess exposure to insulin is the primary cause of insulin resistance and its associated atherosclerosis. Curr Mol Pharmacol. 2011;4:154-66. https://doi.org/10.2174/187446721110403 0154.

26. Hsiang JC, Gane EJ, Bai WW, Gerred SJ. Type 2 diabetes: a risk factor for liver mortality and complications in hepatitis B cirrhosis patients. J Gastroenterol Hepatol. 2015;30:591-9. https://doi.org/10.1111/jgh.12790.

27. Ferrannini E, Cushman WC. Diabetes and hypertension: the bad companions. Lancet. 2012;380:601-10. https://doi.org/10.1016/S0140-6736(12) 60987-8.

28. Schroeder CA Jr, Chen YL, Messina EJ. Inhibition of NO synthesis or endothelium removal reveals a vasoconstrictor effect of insulin on isolated arterioles. Am J Physiol. 1999;276:H815-20. https://doi.org/10.1152/ ajpheart.1999.276.3.H815.

29. Boucher J, Kleinridders A, Kahn CR. Insulin receptor signaling in normal and insulin-resistant states. Cold Spring Harb Perspect Biol. 2014;6:a009191. https://doi.org/10.1101/cshperspect.a009191.

30. Mathur A, Pandey VK, Kakkar P. PHLPP: a putative cellular target during insulin resistance and type 2 diabetes. J Endocrinol. 2017;233:R185-98. https://doi.org/10.1530/JOE-17-0081.

\section{Publisher's Note}

Springer Nature remains neutral with regard to jurisdictional claims in published maps and institutional affiliations.
Ready to submit your research? Choose BMC and benefit from:

- fast, convenient online submission

- thorough peer review by experienced researchers in your field

- rapid publication on acceptance

- support for research data, including large and complex data types

- gold Open Access which fosters wider collaboration and increased citations

- maximum visibility for your research: over 100M website views per year

At BMC, research is always in progress.

Learn more biomedcentral.com/submissions 